\title{
Corrigendum: Vestigial no more
}

\section{Jacques F A P Milller}

Nature Immunology 7, 3-5 (2006); corrected 4 January 2006

In the print version of this essay and the version initially published online, the second sentence of the sixth paragraph is incorrect. It should end “...many questions remained unexamined and unanswered." The error has been corrected in the PDF version of the essay. This correction has been appended to the PDF version. 